\title{
An Inverse Method for The Generation of Random Normal Deviates on Large-Scale Computers
}

\author{
By Mervin E. Muller
}

1. Introduction. Many applications of electronic computers require efficient generation of large numbers of random normal deviates; see, e.g. [7], [12], [14], [17]. Tables of random normal deviates are of course available, for example [18], [23], but they are not sufficiently extensive for many purposes and an outside source of this kind cannot usually be used effectively by the computer. What is required is some method of generation which can be rapidly carried out by the machine itself.

Methods are available by which pseudo random numbers may be produced, see for example [2], [5], [6], [10], [11], [13], [20], [22]. Judging by the results given, for example, by [5], [6], [20], [21], the most satisfactory procedure now in use for generating random numbers is that based on "residue class" techniques, see for example [13], [20]. We shall not consider here the validity of these methods but shall assume that some satisfactory method of producing uniform random deviates is available from which random normal deviates are to be produced. Several ways of generating random normal deviates are known; see, for example, [1], [6], [21], but as is shown in [15], these methods are not as fast nor as reliable, especially for extreme tail values, as that developed here.

In this paper the inverse of the Normal Distribution Function is approximated stepwise by rational functions. Hence a uniform random deviate may be transformed into a normal deviate. The details of the approximations have been obtained for use on a large scale binary machine with index registers, where it is possible to utilize memory space in order to obtain greater speed.

The present approach can be used, if desired, in the course of generating arbitrary $n$-variate normal deviates or Chi-squared variates.

2. Inverse method. In principle, the inverse method of generating a normal deviate $x$ from a uniform deviate $u$ is well known. The problem is to find the inverse relationship $x=x(u)$ given that

$$
u=\frac{1}{\sqrt{2 \pi}} \int_{-\infty}^{x} e^{-t^{2} / 2} d t .
$$

The actual determination of $x(u)$ offers certain numerical difficulties when it is desired to generate reliable normal deviates, especially for large values of $x$. We have considered this problem for two aspects. First, speed and secondly and equally important, reliability. We have insisted that the maximum absolute error in $x$ should be less than $4 \times 10^{-4}$ in the range $-5 \leq x \leq 5$, where we assume

Received October 26, 1957. Prepared in connection with research sponsored by the Office of Ordnance Research, U. S. Army; Statistical Techniques Research Group, Princeton University, Contract No. DA 36-034-ORD 2297. 
$u$ is correct, and where Prob $\{-5 \leq X \leq 5\}>1-6 \times 10^{-7}$ so that $x$ is correct to within $4 \times 10^{-4}$ except for an event of probability less than $6 \times 10^{-7}$.

The relation $x=x(u)$ is approached in stages. The interval $[0,1]$ for $u$ is subdivided so that over each sub-interval it is possible to obtain a reliable and fast procedure for computing $x$. Over most of $(0,1) x=x(u)$ is approximated by Chebyshev polynomials. As $x$ becomes large in absolute value it is necessary to increase the degree of the approximating polynomial. However, even though the degree of the polynomial increases, the frequency with which these approximations are needed decreases; hence this method will use, on the average, a low order approximating polynomial for $x=x(u)$. Due to symmetry, it is actually only necessary to study $x=x(u)$ for $1 / 2 \leq u \leq 1$.

For speed in computing it is best to have the Chebyshev polynomials of as low degree as possible, subject to the specified level of accuracy. Following this approach one could find sub-intervals of greatest or optimum width, see [3]. Two drawbacks then become apparent. If on a binary computer the sub-interval widths are not some negative power of two, considerable computing time is spent in mapping a given sub-interval onto the range $(-1,1)$ which is needed when using a Chebyshev approximation. More important, if the intervals are not of uniform width and a negative power of two, considerable machine time, and memory space, is required to select the appropriate approximation. For, it will not then be possible to use an index register to select the appropriate approximation. Taking these facts into consideration, and taking into account memory space requirements, it was decided to have the widths of the sub-intervals equal $1 / 128$. With this choice of interval width the largest part of $(0,1)$, namely $1 / 8 \leq u \leq 7 / 8$, could be approximated by linear functions. Quadratic and quartic approximations are used for $7 / 8<u \leq 127 / 128$. For $127 / 128<u<1$, and by symmetry $0<u<1 / 128, x=x(u)$ has a singularity of logarithmic type; consequently, for $u$ in this sub-interval an approximation of more subtle type than Chebyshev polynomials is needed.

3. Use of polynomials. Since the techniques used in approximating $x=x(u)$ have possible application when considering the generation of other possible pseudo random variables we shall review the techniques in detail.

To approximate $x=x(u)$ in the $j$ th sub-interval,

$$
\left(\frac{64+j-1}{128} \leq u \leq \frac{64+j}{128}\right), \quad j=1(1) 63,
$$

by Chebyshev polynomials we shall make use of the technique of Interpolating Chebyshev Polynomials as developed by C. Lanczos, see for example [8], [9]. To begin, the $j$ th sub-interval is transformed onto $(-1,1)$ by using

$$
r=256 u-127-2 j \text {. }
$$

Let $r=\cos \theta, 0 \leq \theta \leq \pi$. Let $T_{i}(r)=\cos \left[i\left(\cos ^{-1} r\right)\right]$ denote the $i$ th Chebyshev polynomial. Thus for the $j$ th sub-interval, $x=x_{j}(r)$ will be approximate by a polynomial of $n$th degree, namely,

$$
x_{n j}(r)=1 / 2 d_{0 j}+\sum_{i=1}^{n} d_{i j} T_{i}(r) ;
$$


where the $d_{i j}$ 's are obtained from

$$
d_{i j}=\frac{2}{n+1} \sum_{k=0}^{n} x\left(r_{k}\right) T_{i}\left(r_{k}\right)
$$

and where the $r_{k}$ 's are the zeros of the $(n+1)$ th Chebyshev polynomial. Thus $r_{k}=\cos (\pi(2 k+1) / 2(n+1))$. This approach approximately minimizes the maximum error throughout the sub-interval.

For computing purpose it is more convenient to express the $T_{i}(r)$ 's as polynomials in $r$, see Lanczos [8], [9], for example $T_{1}(r)=r, T_{2}(r)=2 r^{2}-1$. One then obtains

$$
x_{n j}(r)=\sum_{i=0}^{n} a_{i j} r^{i}
$$

The coefficients $a_{i j}$ are given in section 4 .

In calculating the $d_{i j}$ 's, and hence the $a_{i j}$ 's, it is necessary to evaluate $x=x(u)$ for $u_{k j}$ 's where

$$
u_{k j}=\frac{r_{k}+127+2 j}{256}
$$

and this was done for the seven intervals $j=57$ (1)63, i.e., $7 / 8<u \leq 127 / 128$. The values of $x\left(u_{k j}\right)$ were obtained by cubic inverse interpolation in the National Bureau of Standards Tables of the Normal Probability Function, [19].

However, for the intervals, $j=1$ (1)56, i.e., $1 / 2 \leq u \leq 7 / 8$, where the linear approximations are adequate the essential computations for the $a_{i j}$ 's are concerned with finding the inverse values, namely $x=x\left(u_{k j}\right)$. However, for the linear cases the $a_{i j}$ 's given in section 4 were not obtained in the above manner since we had previously needed to have values of $x$ corresponding to $u_{s}=(128+s) / 256$, $s=0,1,2, \cdots, 128$. Consequently we avoided the additional labor of finding the necessary $x$ 's by utilizing the available $x\left(u_{s}\right)$ 's. These values are included as Table 5 in the Appendix. Thus, while not fitting the straight lines by first degree Chebyshev polynomials we were able to obtain the desired level of precision as follows: In the $j$ th interval, where it is appropriate to fit a straight line, consider $u_{j}, u_{j+1}$, and $u_{j, 1 / 2}=\left(u_{j}+u_{j+1}\right) / 2$. Let $x_{j}, x_{j+1}, x_{j, 1 / 2}$ denote the corresponding $x$ values. The $j$ th line is fitted such that the deviations at the ends of the class interval are equal and such that the deviation at the mid-point, namely $u_{j, 1 / 2}$, is equal in magnitude to the deviations at the end points, but is of opposite sign. This approach gives essentially a Chebyshev-type approximation in that we are striving to minimize the maximum error in a given sub-interval. By straightforward computations one then obtains that

$$
\begin{aligned}
& a_{0 j}=\frac{2\left(x_{j+1}+x_{j, 1 / 2}\right)-\left(x_{j+1}-x_{j}\right)(255+4 j)}{4}, \\
& a_{i j}=128\left(x_{j+1}-x_{j}\right) .
\end{aligned}
$$

In the selection of the width of a sub-interval, $w_{j}$, or for determining the appropriate degree of the Chebyshev Polynomial for a given sub-interval, the following 
result is used, see [3] for more general details,

$$
w_{j}=4\left[\frac{(n+1) !}{2} \epsilon\left|x^{(n+1)}\left(c_{j}\right)\right|^{-1}\right]^{\frac{1}{n+1}},
$$

where $\epsilon$ is the largest absolute error that is tolerable, in our case $\epsilon=4 \times 10^{-4}$, and where $x^{(n+1)}(u)$ is the $(n+1)$ st derivative of $x=x(u)$ with respect to $u$.

4. Table of coefficients. The following tables provide the necessary coefficients for the approximations given by equation (1) of section 3 .

\section{TABle 1. Linear Cases}

\begin{tabular}{rrrrrrrrrr}
$j$ & \multicolumn{1}{c}{$a_{0 j}$} & \multicolumn{2}{c}{$a_{1 j}$} & $j$ & $a_{0 j}$ & \multicolumn{2}{c}{$a_{1 j}$} \\
1 & -1.25339 & 449 & 2.50678 & 851 & 29 & -1.56607 & 868 & 2.98461 & 325 \\
2 & -1.25388 & 344 & 2.50775 & 044 & 30 & -1.59698 & 101 & 3.02714 & 356 \\
3 & -1.25487 & 723 & 2.50967 & 688 & 31 & -1.63039 & 945 & 3.07264 & 746 \\
4 & -1.25639 & 365 & 2.51257 & 300 & 32 & -1.66656 & 010 & 3.12136 & 699 \\
5 & -1.25845 & 203 & 2.51644 & 669 & 33 & -1.70571 & 765 & 3.17357 & 468 \\
6 & -1.26107 & 329 & 2.52130 & 843 & 34 & -1.74815 & 989 & 3.22957 & 838 \\
7 & -1.26428 & 016 & 2.52717 & 152 & 35 & -1.79421 & 327 & 3.28972 & 698 \\
8 & -1.26809 & 727 & 2.53405 & 216 & 36 & -1.84424 & 956 & 3.35441 & 736 \\
9 & -1.27255 & 126 & 2.54196 & 946 & 37 & -1.89869 & 401 & 3.42410 & 298 \\
10 & -1.27767 & 105 & 2.55094 & 571 & 38 & -1.95803 & 516 & 3.49930 & 401 \\
11 & -1.28348 & 794 & 2.56100 & 644 & 39 & -2.02283 & 720 & 3.58062 & 028 \\
12 & -1.29003 & 594 & 2.57218 & 075 & 40 & -2.09375 & 508 & 3.66874 & 687 \\
13 & -1.29735 & 187 & 2.58450 & 135 & 41 & -2.17155 & 347 & 3.76449 & 389 \\
14 & -1.30547 & 572 & 2.59800 & 495 & 42 & -2.25713 & 085 & 3.86881 & 137 \\
15 & -1.31445 & 096 & 2.61273 & 256 & 43 & -2.35154 & 997 & 3.98282 & 083 \\
16 & -1.32432 & 485 & 2.62872 & 974 & 44 & -2.45607 & 734 & 4.10785 & 604 \\
17 & -1.33514 & 882 & 2.64604 & 704 & 45 & -2.57223 & 456 & 4.24551 & 603 \\
18 & -1.34698 & 984 & 2.66475 & 763 & 46 & -2.70077 & 415 & 4.39645 & 556 \\
19 & -1.35986 & 540 & 2.68485 & 495 & 47 & -2.84833 & 659 & 4.56815 & 521 \\
20 & -1.37390 & 816 & 2.70651 & 014 & 48 & -3.01223 & 276 & 4.75713 & 372 \\
21 & -1.38914 & 726 & 2.72973 & 042 & 49 & -3.19590 & 941 & 4.96703 & 562 \\
22 & -1.40567 & 396 & 2.75461 & 642 & 50 & -3.40829 & 673 & 5.20760 & 458 \\
23 & -1.42357 & 635 & 2.78126 & 053 & 51 & -3.65403 & 652 & 5.48350 & 516 \\
24 & -1.44295 & 134 & 2.80976 & 489 & 52 & -3.93953 & 404 & 5.80124 & 828 \\
25 & -1.46390 & 578 & 2.84024 & 264 & 53 & -4.27256 & 248 & 6.16869 & 364 \\
26 & -1.48655 & 769 & 2.87281 & 914 & 54 & -4.67044 & 630 & 6.60394 & 516 \\
27 & -1.51103 & 773 & 2.90763 & 359 & 55 & -5.15058 & 375 & 7.12472 & 205 \\
28 & -1.53749 & 093 & 2.94484 & 078 & 56 & -5.74560 & 472 & 7.76467 & 397
\end{tabular}

TABLE 2. Quadratic Cases

\begin{tabular}{|c|c|c|c|}
\hline$j$ & $a_{0 j}$ & $a_{1 j}$ & $a_{2 j}$ \\
\hline 57 & 1.56668859 & -0.0334348405 & .000875575 \\
\hline 58 & 1.63732538 & $-0.03745 \quad 15701$ & .00114804 \\
\hline 59 & 1.71722812 & -0.0428426652 & .00157546 \\
\hline 60 & 1.80989233 & -0.0504900254 & .00230549 \\
\hline 61 & 1.92135077 & -0.0622630013 & .00372027 \\
\hline 62 & 2.06352790 & -0.0829931005 & .00708977 \\
\hline
\end{tabular}

TABLE 3. Quartic Case

$$
\begin{aligned}
& \begin{array}{ccccc}
j & a_{0 j} & a_{1 j} & a_{2 j} & a_{3 j}
\end{array} \\
& 63+2.26622681+0.12757931+0.01844432+0.0042442872+0.0010406032
\end{aligned}
$$


5. The interval $(127 / 128<u<1)$. In view of the fact that $x(u)$ may be looked upon as a function having a singularity of logarithmic type in this interval, it is necessary to abandon the use of a polynomial type approximation here. A satisfactory rational approximation is obtained by using a truncated continued fraction expansion, see for example [4], [16]. For any given value of $u$ the corresponding value of $x$ is approximated by the following recurrence relation. The $k$ th convergent to $x(u)$ is given by

$$
x_{k}(u)=\frac{M_{k}(u)}{N_{k}(u)},
$$

where $M_{k}(u)$ and $N_{k}(u)$ are determined as follows:

$$
\begin{aligned}
& M_{k+1}(u)=d_{k} M_{k}(u)+\left(u-u_{k-1}\right) M_{k-1}(u) ; \quad M_{0}(u)=1 ; \quad M_{1}(u)=d_{0} ; \\
& N_{k+1}(u)=d_{k} N_{k}(u)+\left(u-u_{k-1}\right) N_{k-1}(u) ; \quad N_{0}(u)=0 ; \quad N_{1}(u)=1 ;
\end{aligned}
$$

and where $d_{k}$ denotes a selected value of the $k$ th inverted divided difference of $x(u)$.

In order to insure sufficient precision it is suggested that the machine program test to see if $u>.99999$. When $u$ is greater than .99999 it is suggested that the additional significant figures for $u_{k}, k=10(1) 14$, be utilized. Double precision operations are not necessary if these $u_{k}$ are stored with the first three nines suppressed, $u$ must then be shifted the appropriate number of places before performing $u-u_{k}$.

Following [4], page 406, $x_{k}(u)$ can be computed from

$$
x_{k}(u)=d_{0}+\sum_{n=1}^{k} \frac{(-1)^{n+1}\left(u-u_{0}\right)\left(u-u_{1}\right) \cdots\left(u-u_{n-1}\right)}{N_{n}(u) N_{n+1}(u)} .
$$

To insure that $x(u)$ can be approximated to within $4 \times 10^{-4}$ it is necessary to stop at an appropriate value of $k=k(u)$ since the approximation being employed is essentially a divergent expansion. Numerical evaluations verified that it is

\begin{tabular}{|c|c|c|}
\hline$k$ & $u_{k}$ & $d_{k}$ \\
\hline $\mathbf{0}$ & .9922397464 & 2.420000000 \\
\hline 1 & .9946138540 & 0.018262366 \\
\hline 2 & .9965330262 & -0.655181080 \\
\hline 3 & .9981341867 & 0.023997757 \\
\hline 4 & .9990323968 & -0.267371460 \\
\hline 5 & .9993128620 & 0.016541263 \\
\hline 6 & .9996630707 & -0.141949840 \\
\hline 7 & .9998408914 & 0.0099732778 \\
\hline 8 & .9999276519 & -0.060049158 \\
\hline 9 & .9999683287 & 0.0051181541 \\
\hline 10 & $.99998 \quad 66542 \quad 51$ & -0.023299296 \\
\hline 11 & $.9999945874 \quad 56$ & 0.0024107770 \\
\hline 12 & $.9999978875 \quad 45$ & -0.0086334192 \\
\hline 13 & .999999206672 & 0.0010076316 \\
\hline & $\begin{array}{ccc}99999 & 97133 \quad 484\end{array}$ & $-0.00308 \quad 28145$ \\
\hline
\end{tabular}
sufficient to have the machine program terminate as soon as one of the following three conditions is satisfied, namely: (i) select $x_{k}(u)$ as the approximation to $x(u)$

TABLE 4. Constants for Rational Approximation 
if $r_{k}=\left|x_{k}(u)-x_{k-1}(u)\right|<4 \times 10^{-4}$, (ii) select $x_{k}(u)$ as the approximation to $x(u)$ if $r_{k}-r_{k+1}<0$, and (iii) if $k=14$ terminate the process and select $x_{14}(u)$ as the approximation to $x(u)$.

The value of $k$ increases as $u$ increases in the interval $(127 / 128<u<1$ $-3 \times 10^{-7}$ ), and consequently so does the computing time. However, this subinterval will, on the average, increase the necessary computing time very little. It should be kept in mind that while this method is very appropriate and convenient for a machine which performs "floating point" multiplication, this approach would create serious scaling difficulties for a "fixed point" mode of operation.

6. Concluding comments. A detailed comparison of this method with other known methods is given in [15]. The technique proposed in this paper yields accuracy comparable with, or better than, most previous proposals using about one-quarter the computing time while requiring about twice as many memory locations.

The author wishes to express his appreciation to Dr. G. E. P. Box, Dr. Collin Mallows, and Dr. John W. Tukey for their interest and generous comments concerning this work. The author is also indebted to Mrs. A. Schay for her excellent help with the numerical computations.

Princeton University

Princeton, New Jersey

1. G. E. P. Box \& M. E. Muller, "A note on the generation of normal deviates," $A n n$. Math. Stat., to be published.

2. GEORGE E. FORSYTHE, "Generation and testing of random digits at the National Bureau of Standards, Los Angeles," Monte Carlo Method, NBS, Applied Mathematics Series 12, U. S. Government Printing Office, Washington, D. C., 1951, p. 34-35. [MTAC, Rev. 42, v. XI, 1957, p. $43-44$.

3. JOSEPH O. HARRISON, JR., "Piecewise polynomial approximations for large-scale digital calculations." [MTAC, v. III, 1949, p. 400-407.]

4. F. B. HILDEBRAND, Introduction to Numerical Analysis, McGraw-Hill Book Co., Inc., New York, 1956.

5. D. L. JoHNSON, "Generating and testing pseudo random numbers on the IBM type 701," $M T A C$, v. X, 1956, p. 8-13.

6. M. L. JuNCOSA, "Random number generation on the BRL High-Speed computing machines," Report 855, Ballistic Research Laboratories, Aberdeen Proving Ground, Maryland, 1953.

7. Herman KaHN, "Applications of Monte Carlo," RAND Project Report, 1954, Revised 1956, RAND Project Report RM-1237-AEC.

8. C. LANczos, "Trigonometric interpolation of empirical and analytical functions," Jn. Math. Phys., v. 17, 1938, p. 123-199.

9. C. Lanczos, Tables of Chebyshev Polynomials $S_{n}(x)$ and $C_{n}(x)$, NBS, Applied Mathematics Series 9, U. S. Government Printing Office, Washington, D. C., 1952. [MTAC, Rev. 1103, v. 7, 1953, p. 174.]

10. D. H. LeHMER, "Mathematical methods in large scale computing units," Proceedings Second Symposium on Large-Scale Digital Calculating Machinery, 1949, p. 141-146. Harvard University Press, Cambridge, Mass., 1951.

11. D. H. LEHMER, Description of "Random number generation of the BRL high-speed computing machines," Mathematical Reviews, v. 15, 1954, p. 559.

12. NBS, Applied Mathematics Series 12, Monte Carlo Methods, 1951, p. 34-35.

13. J. MoshmaN, "The generation of pseudo-random numbers on a decimal calculator," Jn., Assoc. for Computing Machinery, v. 1, 1954, p. 88-91.

14. M. E. MULLER, "Some continuous Monte Carlo methods for the Dirichlet problem," Ann. Math. Stat., v. 27, p. 569-589.

15. M. E. MuLLER, "A comparison of methods for generating normal deviates," Technical Report No. 9, Statistical Techniques Research Group, Department of Mathematics, Princeton University, to be published.

16. N. E. NORLUND, Vorlesungen Uber Differenzenrechnung, Springer, Berlin, 1924.

17. HERBERT A. MEYER, Editor, Symposium on Monte Corlo Methods, held at the University of Florida, 1954, John Wiley and Sons, Inc., New York, 1956. [MTAC, Rev. 43, v. XI, 1957, p. 44-46.]

18. The Rand Corporation, One Million Random Digits and 100,000 Normal Deviates, The Free Press, Glencoe, Illinois, 1955. [MTAC, Rev. 11, v. X, 1956, p. 39-43.] 
19. NBS, Applied Mathematics Series 23, Tables of Normal Probability Functions, U. S. Government Printing Office, Washington, D. C., 1953.

20. O. TAUSSKY \& J. ToDD, "Generation and testing of pseudo-random numbers," Symposium on Monte Carlo Methods, University of Florida, 1954, John Wiley and Sons, Inc., 1956, p. 15-28.

21. D. TeIchroew, Distribution Sampling with High-Speed Computers, Ph.D. Thesis, University of North Carolina, 1953.

22. D. F. Votaw, JR. \& J. A. Rafferty, "High speed sampling," $M T A C$, v. 5, 1951, p. 1-8.

23. H. WolD, "Random normal deviates," Tracts for Computers, No. XXV, Cambridge University Press, New York, 1948.

\section{Appendix}

This table gives values of $x(u)$ corresponding to the Normal Distribution for $F\left(x_{j}\right)=1 / 2+j / 256, j=1(1) 127$. The values were obtained from the National Bureau of Standard Tables of the Normal Probability Function, [19], by using the inversion formula:

$$
x(u)=u_{0}+\frac{a}{2 Q_{0}}+\frac{u_{0}}{2}\left(\frac{a}{2 Q_{0}}\right)^{2}+\frac{\left(2 u_{0}^{2}+1\right)}{6}\left(\frac{a}{2 Q_{0}}\right)^{3},
$$

where $u_{0}$ is the nearest tabulated entry to $u$, and $u-u_{0}=a$, and where

$$
Q_{0}=\frac{1}{\sqrt{2 \pi}} e^{-\left[x\left(u_{0}\right)\right]^{2} / 2} .
$$

\begin{tabular}{|c|c|c|c|c|c|}
\hline$j$ & $F\left(x_{j}\right)=1 / 2+j / 256$ & $x_{j}$ & $j$ & $F\left(x_{j}\right)=1 / 2+j / 256$ & $x_{j}$ \\
\hline 1 & 0.50390625 & 0.00979167 & 33 & 0.62890625 & 0.32895791 \\
\hline 2 & 0.50781250 & $0.01958 \quad 429$ & 34 & 0.63281250 & 0.33931161 \\
\hline 3 & 0.51171875 & 0.02937878 & 35 & 0.63671875 & $0.34970 \quad 180$ \\
\hline 4 & 0.51562500 & 0.03917609 & 36 & 0.64062500 & 0.36013003 \\
\hline 5 & 0.51953125 & 0.04897716 & 37 & $0.64453 \quad 125$ & 0.37059729 \\
\hline 6 & 0.52343750 & 0.05878294 & 38 & 0.64843750 & $0.38110 \quad 545$ \\
\hline 7 & 0.52734375 & 0.06859437 & 39 & 0.65234375 & $0.39165 \quad 587$ \\
\hline 8 & 0.53125000 & 0.07841241 & 40 & 0.65625000 & 0.40225007 \\
\hline 9 & 0.53515625 & 0.08823802 & 41 & 0.66015625 & 0.41288960 \\
\hline 10 & 0.53906250 & $0.09807 \quad 215$ & 42 & 0.66406250 & $0.42357 \quad 608$ \\
\hline 11 & 0.54296875 & $0.10791 \quad 578$ & 43 & 0.66796875 & $0.43431 \quad 116$ \\
\hline 12 & 0.54687500 & 0.11776987 & 44 & 0.67187500 & 0.44509652 \\
\hline 13 & $0.55078 \quad 125$ & 0.12763542 & 45 & $0.67578 \quad 125$ & 0.45593392 \\
\hline 14 & 0.55468750 & 0.13751340 & 46 & 0.67968750 & 0.46682512 \\
\hline 15 & 0.55859375 & $0.14740 \quad 482$ & 47 & 0.68359375 & 0.47777199 \\
\hline 16 & $0.56250 \quad 000$ & $\begin{array}{lll}0.15731 & 068\end{array}$ & 48 & $0.68750 \quad 000$ & $0.48877 \quad 641$ \\
\hline 17 & $0.56640 \quad 625$ & 0.16723201 & 49 & $0.69140 \quad 625$ & $984 \quad 034$ \\
\hline 18 & 0.57031250 & 16982 & 50 & 0.69531250 & 0.5109658 \\
\hline 19 & 0.57421875 & 0.18712516 & 51 & 0.69921875 & $0.52215 \quad 488$ \\
\hline 20 & 0.57812500 & 0.19709908 & 52 & 0.70312500 & $0.53340 \quad 971$ \\
\hline 21 & $0.58203 \quad 125$ & 0.20709265 & 53 & 0.70703125 & 0.54473251 \\
\hline 22 & 0.58593750 & 0.21710695 & 5 & 0.71093750 & 0.55612559 \\
\hline 23 & 0.58984375 & 0.22714306 & 55 & 0.71484375 & $0.56759 \quad 132$ \\
\hline 2 & 0.59375000 & $0.23720 \quad 211$ & 56 & 875000 & 1321 \\
\hline 25 & $0.59765 \quad 625$ & 0.24728522 & 57 & 0.72265625 & 0.59075066 \\
\hline 2 & 0.60156250 & 0.25739353 & 58 & 0.72656250 & 0.60244945 \\
\hline 2 & 0.60546875 & 0.26752821 & 59 & 0.73046875 & $0.61423 \quad 129$ \\
\hline 28 & 0.60937500 & $\begin{array}{lll}0.27769 & 044\end{array}$ & 60 & 0.73437500 & 0.62609901 \\
\hline 2 & $0.61328 \quad 125$ & $0.28788 \quad 143$ & 61 & $0.73828 \quad 125$ & 0.63805558 \\
\hline 3 & 0.61718750 & 0.29810241 & 62 & 0.74218750 & 0.6501040 \\
\hline & $\begin{array}{lll}0.62109 & 37\end{array}$ & $0.30835 \quad 463$ & 63 & $0.74609 \quad 375$ & 0.6622476 \\
\hline & 0.625 & 0.31863 & & $0.75000 \quad 000$ & 0.67448 \\
\hline
\end{tabular}

TABLE 5. Inverse Values for the Normal Distribution 
TABLE 5-Continued

\begin{tabular}{|c|c|c|c|c|c|}
\hline$j$ & $F\left(x_{j}\right)=1 / 2+j / 256$ & $x_{j}$ & $j$ & $F\left(x_{j}\right)=1 / 2+j / 256$ & $x_{j}$ \\
\hline 65 & 0.75390625 & $0.68683 \quad 375$ & 97 & 0.87890625 & 1.16953661 \\
\hline 66 & 0.75781250 & 0.69928330 & 98 & 0.88281250 & 1.18916435 \\
\hline 67 & 0.76171875 & 0.71184220 & 99 & 0.88671875 & 1.20926123 \\
\hline 68 & 0.76562500 & $0.72451 \quad 438$ & 100 & 0.89062500 & 1.22984876 \\
\hline 69 & 0.76953125 & 0.73730400 & 101 & $0.89453 \quad 125$ & $1.25099 \quad 172$ \\
\hline 70 & 0.77343750 & 0.75021538 & 102 & 0.89843750 & 1.27268865 \\
\hline 71 & 0.77734375 & $\begin{array}{lll}0.76325 & 304\end{array}$ & 103 & 0.90234375 & 1.29502241 \\
\hline 72 & 0.78125000 & 0.77642176 & 104 & 0.90625000 & 1.31801090 \\
\hline 73 & $0.78515 \quad 625$ & 0.78972652 & 105 & 0.91015625 & 1.34171784 \\
\hline 74 & 0.78906250 & $\begin{array}{lll}0.80317 & 257\end{array}$ & 106 & 0.91406250 & 1.36620382 \\
\hline 75 & 0.79296875 & 0.81676542 & 107 & 0.91796875 & 1.39153749 \\
\hline 76 & 0.79687500 & 0.83051088 & 108 & 0.92187500 & 1.41779714 \\
\hline 77 & $0.80078 \quad 125$ & 0.84441508 & 109 & $0.92578 \quad 125$ & $1.44507 \quad 258$ \\
\hline 78 & 0.80468750 & $0.85848 \quad 447$ & 110 & 0.92968750 & 1.47345903 \\
\hline 79 & 0.80859375 & 0.87272589 & 111 & 0.93359375 & 1.50310294 \\
\hline 80 & 0.81250000 & 0.88714656 & 112 & 0.93750000 & 1.53412054 \\
\hline 81 & $0.81640 \quad 625$ & 0.90175411 & 113 & 0.94140625 & 1.56668859 \\
\hline 82 & 0.82031250 & 0.91655667 & 114 & 0.94531250 & 1.60100866 \\
\hline 83 & 0.82421875 & 0.93156283 & 115 & 0.94921875 & 1.63732538 \\
\hline 84 & 0.82812500 & 0.94678176 & 116 & 0.95312500 & 1.67594192 \\
\hline 85 & $0.83203 \quad 125$ & 0.96222320 & 117 & $0.95703 \quad 125$ & 1.71722812 \\
\hline 86 & 0.83593750 & 0.97789754 & 118 & 0.96093750 & 1.76167041 \\
\hline 87 & 0.83984375 & 0.99381591 & 119 & 0.96484375 & 1.80989233 \\
\hline 88 & 0.84375000 & 1.00999017 & 120 & 0.96875000 & 1.86273187 \\
\hline 89 & $0.84765 \quad 625$ & 1.02643306 & 121 & 0.97265625 & 1.92135077 \\
\hline 90 & 0.85156250 & 1.04315826 & 122 & 0.97656250 & 1.98742789 \\
\hline 91 & 0.85546875 & 1.06018048 & 123 & 0.98046875 & 2.06352790 \\
\hline 92 & 0.85937500 & 1.07750557 & 124 & 0.98437500 & 2.15387469 \\
\hline 93 & $0.86328 \quad 125$ & 1.09518065 & 125 & $0.98828 \quad 125$ & 2.26622681 \\
\hline 94 & 0.86718750 & 1.11319428 & 126 & 0.99218750 & 2.41755902 \\
\hline 95 & 0.87109375 & 1.13157656 & 127 & $0.99609 \quad 375$ & 2.66006747 \\
\hline 96 & 0.87500000 & 1.15035938 & & & \\
\hline
\end{tabular}

\title{
Algebraic Approximations for Laplace's Equation in the Neighborhood of Interfaces
}

\author{
By J. W. Sheldon
}

1. Introduction. Let it be required to solve the following problem:

Problem A:

Let $C_{i}, i=1,2$, be two simple, closed plane curves with continuous curvature. Let $C_{2}$ enclose all the points of $C_{1}$. Let $G_{1}$ be the region interior to $C_{1}$. Let $G_{2}$ be the region interior to $C_{2}$ and exterior to $C_{1}$. Let $W$ be a continuous, bounded function of position on $C_{2}$. Let it be required to find harmonic functions $V^{(i)}$ such that

(a) $V^{(2)}=W$ on $C_{2}$.

(b) $V^{(i)}$ is regular and bounded in $G_{i}$.

Received September 19, 1957. 